\title{
Valuation of a new Cromogenic Agar Medium for the detection of ESBL producing Enterobacteriaceae
}

\author{
Carlo Mancini, Daniela Danieli, Adrio Penni, Marta Mingione, Fabrizio Tosto, \\ Gianluca Puggioni, Paola Varesi, Alessandra Giordano \\ U.O.C. Analisi Microbiologiche B Azienda Policlinico Umberto IV.le del Policlinico I55 00 I6I Roma
}

Key words: Cromogenic agar medium, ESBL, Enterobacteriaceae

Valutazione di un nuovo terreno cromogeno selettivo per la presenza di enterobatteri produttori di ESBL

\section{SUMMARY}

Escherichia coli producing ESBL are a major problem in the many different hospitals worldwide causing outbreaks as well as sporadic infections.

A novel chromogenic agar medium (ChromID ESBL bioMèrieux, Marcy L'Etoile, France) was tested directly on a total of 162 clinical samples and plated in parallel on to common media including Mac Conkey agar (bioMèrieux, Marcy L'Etoile, France). Colonies presenting a different morphologic aspect and growing on media were identified and tested for susceptibility by VITEK 2 using GN-cards and AST-NOI 3 cards (bioMérieux, Marcy L'Etoile, France) Confirmation of ESBL-producing isolates was performed by combinated double disk.

We are not find errors. The chromID ESBL appears as an excellent medium for the screening and presumptive identification of ESBLproducing Enterobacteriaceae directly from clinical samples. Further studies with a wider range of clinical specimens are required to confirm this utility.

\section{INTRODUZIONE}

Nell'ambito delle patologie infettive ad eziologia batterica, le problematiche emergenti sono legate soprattutto allo sviluppo di antibioticoresistenza in importanti patogeni.

Il problema che è sorto con l'introduzione in terapia delle prime molecole antimicrobiche, è drammaticamente peggiorato negli ultimi due decenni interessando gran parte delle aree del pianeta (1).

Per quanto riguarda i microrganismi Gramnegativi le problematiche emergenti sono rappresentate dalla diffusione massiccia in alcune specie delle Enterobacteriaceae delle beta-lattamasi a spettro esteso (ESBL), enzimi in grado di idrolizzare le più potenti cefalosporine, dalla descrizione delle prime carbapenemasi, enzimi con attività inattivante nei confronti dei carbapenemici, considerati farmaci di ultima risorsa nei confronti degli stipiti produttori di ESBL e dalla diffusione di stipiti di Pseudomonas spp. multiresistenti. In Italia $i$ dati più recenti stimano l'incidenza in ambito nosocomiale di ceppi produttori di ESBL intorno al 15-30\%, con Klebsiella spp., Proteus spp. ed E. coli le specie più coinvolte $(3,6)$ e oltre il $20 \%$ di Pseudomonas aeruginosa simultaneamente resistenti a beta-lattamici, fluorochinoloni e aminoglicosidi.

Il presente studio ha voluto valutare la specificità e il valore predittivo delle piastre chromID ESBL (bioMérieux), un nuovo terreno cromogeno selettivo per lo screening degli enterobatteri produttori di $\operatorname{ESBL}(2,4)$.

\section{MATERIALI E METODI}

162 campioni clinici (destinati alla routine diagnostica) provenienti da reparti ad alto rischio quali Rianimazione, Trapianti, Neurochirurgia e Cardiochirurgia sono stati seminati sui comuni terreni di coltura e, una aliquota residua, sul "ChromID ESBL medium" (bioMérieux).

Sui ceppi di enterobatteri isolati dalla piastra di "ChromID ESBL medium", a conferma del risultato ottenuto con la piastra cromogena, gli isolati sono stati caratterizzati per specie e per fenotipo di resistenza mediante il sistema VITEK 2 (card ID-GN e card AST-N041 bioMérieux) ed eseguita la conferma mediante test del doppio disco per diffusione (5). 
Tabella I. Ceppi isolati da chromID agar

\begin{tabular}{|c|c|c|c|c|c|c|c|}
\hline DATA & CAMPIONE & REPARTO & MATERIALE & COLORE & SPECIE & VITEK & KB \\
\hline $26 / 05 / 2007$ & 60665 & BANOI & EMO & Verde & K. pneumoniae & ESBL+ & POS \\
\hline $08 / 05 / 2007$ & 59876 & BANOI & T.Ferita & Verde & K. pneumoniae & ESBL+ & POS \\
\hline $18 / 05 / 2007$ & 4392 & BAN05 & TAS & Verde & K. pneumoniae & ESBL+ & POS \\
\hline $26 / 04 / 2007$ & 3998 & BAN05 & Urine & Verde & K. pneumoniae & ESBL+ & POS \\
\hline $30 / 04 / 2007$ & 4077 & BAN05 & Urine & Rosa scuro & E. coli & ESBL+ & POS \\
\hline $30 / 04 / 2007$ & 4078 & BAN05 & T.Ferita & Rosa scuro & E. coli & ESBL+ & POS \\
\hline $30 / 04 / 2007$ & 4079 & BAN05 & TAS & Verde & K. pneumoniae & ESBL+ & POS \\
\hline $30 / 04 / 2007$ & 4056 & BAN05 & EMO & Verde & K. pneumoniae & ESBL+ & POS \\
\hline $25 / 05 / 2007$ & 60630 & BCGIO & Liq Biliare & Verde & E. cloacae & ESBL+ & POS \\
\hline $20 / 04 / 2007$ & 59275 & BCGIO & Urine & BIANCHE & E. coli & ESBL+ & NEG \\
\hline $05 / 05 / 2007$ & 59786 & BCGIO & Urine & Rosa scuro & E. coli & ESBL+ & POS \\
\hline $05 / 05 / 2007$ & 59776 & BCGIO & Urine & Rosa scuro & E. coli & ESBL+ & POS \\
\hline $02 / 05 / 2007$ & 4092 & $\mathrm{BMClO}$ & Urine & Verde-blu & E. cloacae & ESBL+ & POS \\
\hline $15 / 05 / 2007$ & 4325 & BMCIO & Liq Ascesso & Verde-blu & E. cloacae & ESBL+ & POS \\
\hline $23 / 04 / 2007$ & 3968 & $\mathrm{BMClO}$ & T.Vaginale & Rosa scuro & E. coli & ESBL + & POS \\
\hline $21 / 05 / 2007$ & 60348 & $\mathrm{BMClO}$ & Urine & Rosa scuro & E. coli & ESBL+ & POS \\
\hline
\end{tabular}

Tabella 2. Risultati della valutazione dei campioni clinici REPARTI CAMPIONI chromID VITEK2 KB \% ESBL POS

\begin{tabular}{lccccc}
\hline RIANIMAZIONE & & & & & \\
BAN0I & 59 & 2 & 2 & 2 & 3.40 \\
\hline $\begin{array}{l}\text { RIAN/NEURO } \\
\text { BAN05 }\end{array}$ & 29 & 6 & 6 & 6 & 20.7 \\
\hline $\begin{array}{l}\text { NEUROCHIR. } \\
\text { BAN05 }\end{array}$ & 10 & 0 & 0 & 0 & 0 \\
\hline $\begin{array}{l}\text { CADIOCHIR. } \\
\text { BMCI0 }\end{array}$ & 24 & 4 & 4 & 4 & 16.70 \\
\hline $\begin{array}{l}\text { TRAPIANTI } \\
\text { BCGI0 }\end{array}$ & 40 & 3 & 4 & 3 & 7.50 \\
\hline
\end{tabular}

\section{RISULTATI}

Sono stati valutati 59 campioni dal reparto di Rianimazione e Terapia Intensiva, 29 dalla Terapia Intensiva Neurochirurgica, 10 dalla Neurochirurgia, 24 dalla Cardiochirurgia e 40 dalla Chirurgia dei Trapianti d'Organo. Sul terreno dopo $24 \mathrm{~h}$ di incubazione si è evidenziata la crescita di 15 sospetti Enterobatteri produttori di ESBL (tabelle 1, 2). Nel resoconto dell'AES del Vitek2 si è trovato un ceppo di E. coli ESBL positivo mentre sul chromID si sono evidenziate colonie bianche (ESBL-). Con la tecnica del doppio disco questo ceppo non ha mostrato alcun sinergismo indicando pertanto la presenza di un ceppo non produttore di ESBL.

\section{CONCLUSIONI}

Utilizzando, inserito nella routine, il ChromoID ESBL abbiamo potuto valutare l'attendibilità di questa piastra di screening.

1. Non abbiamo evidenziato discordanze.

2. È utile anche dal punto di vista infettivologico perché evidenzia la presenza di ceppi che presentano multiresistenze, infatti le altre specie batteriche cresciute sul terreno ( $P$. aerugino$s a$, A. baumannii, etc.) presentavano tutte mul- tirestenze.

L'utilizzo del terreno chromID ESBL è un buon screening per evidenziare rapidamente la presenza di Enterobatteri produttori di ESBL e/o multiresistenze permettendoci di prevenire e/o combattere le infezioni ospedaliere rilevando la presenza di pazienti colonizzati o infettati in sole $24 \mathrm{~h}$.

È nostra intenzione continuare lo studio su quantità maggiore di campioni e sui ceppi isolati lo studio proseguirà con l'utilizzo della biologia molecolare per decodificare gli enzimi di resistenza.

\section{BIBLIOGRAFIA}

1. Bradford PA. Extended-spectrum beta-lactamase in the 21st century: characterization, epidemiology, and detection of this important resistance threat. Clin Microbiol Rev 2001; 14: 933-51.

2. Ciragil P, Gul M, Aral M, Ekerbicer H. Evaluation of a new chromogenic medium for isolation and identification of common urinary tract pathogens. Eur J Clin Microbiol Infect Dis 2006; 25: 108-11.

3. Du B, Long Y, Liu H, Chen D, Liu D, Xu Y, Xie X. Extendedspectrum-\{beta\}-lactamase-producing Escherichia coli and Klebsiella pneumoniae bloodstream infection: risk factors and clinical outcome. Intensive Care Med 2002; 28: 1718-23.

4. Glupczynski Y, Berhin C, Bauraing C, Bogaerts P. Evaluation of a new selective chromogenic agar medium for detection of extended-spectrum beta-lactamase-producing Enterobacteriaceae.J Clin Microbiol 2007; 45(2): 501-5.

5. Jarlier V, Nicolas MH, Fournier G, Philippon A. Extended broad-spectrum ß-lactamases conferring transferable resistance to newer $\beta$-lactam agents in Enterobacteriaceae: hospital prevalence and susceptibility pattern. Rev Infect Dis 1988; 10: 867-78.

6. Lautenbach E, Patel JB, Bilker WB, Edelstein PH, Fishman NO. Extended-spectrum-\{beta $\}$-lactamaseproducing Escherichia coli and Klebsiella pneumoniae: risk factors for infection and impact of resistance on outcomes. Clin Infect Dis 2001; 32: 1162-71. 